\title{
A two-stage classification algorithm for radar targets based on compressive detection
}

\author{
Cong Liu ${ }^{1} \mathbb{D}$, Yunqing Liu ${ }^{1,2^{*}}$, Qiong Zhang ${ }^{1}$, Xiaolong Li ${ }^{1}$, Tong Wu ${ }^{1}$ and Qi Li ${ }^{1}$
}

\author{
*Correspondence: mzliuyunqing@ \\ 163.com \\ ${ }^{1}$ School of Electronic and \\ Information Engineering, \\ Changchun University of Science \\ and Technology, Changchun, Jilin \\ 130022, China \\ ${ }^{2}$ Institute of Space Optical Electronic \\ Technology, Changchun University \\ of Science and Technology, \\ Changchun, Jilin 130022, China
}

\begin{abstract}
Algorithms are proposed to address the radar target detection problem of compressed sensing (CS) under the conditions of a low signal-to-noise ratio (SNR) and a low signal-to-clutter ratio (SCR) echo signal. The algorithms include a twostage classification for radar targets based on compressive detection (CD) without signal reconstruction and a support vector data description (SVDD) one-class classifier. First, we present the sparsity of the echo signal in the distance dimension to design a measurement matrix for CD of the echo signal. Constant false alarm rate (CFAR) detection is performed directly on the CD echo signal to complete the firstorder target classification. In simulations, the detection performance is similar to that of the traditional matched filtering algorithm, but the data rate is lower, and the necessary data storage space is reduced. Then, the power spectrum features are extracted from the data after the first-order classification and converted to the feature domain. The SVDD one-class classifier is introduced to train and classify the characteristic signals to complete the separation of the targets and the false alarms. Finally, the performance of the algorithm is verified by simulation. The number of false alarms is reduced, and the detection probability of the targets is improved.

Keywords: Compressive detection, CFAR, Power spectrum feature, Two-stage classification
\end{abstract}

\section{Introduction}

Modern radar is being developed with increasingly wide bandwidth and high resolution [1]. These systems generate large amounts of data, require more storage space, and necessitate a higher level of data processing [2]. According to the classic Nyquist sampling theorem, if undistorted reconstruction is performed on the signal, the sampling frequency of the system must be at least twice the highest frequency of the sampled signal; this is a challenge for real-time signal processing [3]. The proposed theory of compressed sensing (CS) [4,5] addresses the traditional Nyquist problems [6]. This theory has been widely used in the field of radar signal processing

The radar target signal is sparse. According to the CS theory, when the signal is sparse or compressible, solving an optimization problem can accurately or 
approximately reconstruct the original signal from low-dimensional observation data [10]. Current signal reconstruction methods include the greedy algorithm [11, 12], orthogonal matching pursuit [13, 14], basis pursuit [4, 15], and other methods [16-18]. However, the process of signal reconstruction is difficult and involves complex iterative algorithms that increase the amount of data. Therefore, a new signal processing method based on compressive detection (CD) has attracted increasing attention [19-24].

Radar experiences interference from clutter in the process of detecting a target. A constant false alarm rate (CFAR) detector adaptively changes the detection threshold as the reference cell changes to achieve CFAR detection of targets [25]. Extensive radar target detection results have been achieved with CFAR [26-28]. In radar target recognition, sparse representation research focuses on extracting accurate target feature information from the received echo data [29]. Combining CS with traditional CFAR detection has been a research trend for target detection [30, 31]. In [30] and [31], the compressed signal is reconstructed first, and cell average CFAR (CA-CFAR) detection is then performed. However, the reconstruction of the signal requires a high signal-tonoise ratio (SNR). For the signal detection, it is necessary to determine only whether the target exists; it is not necessary to perform the detection after the signal is reconstructed [22, 32-35]. In [22, 32, 34], compressive detection algorithms based on traditional matched filter methods were proposed. For unknown parameter signal detection, providing all the signal information before detection is inefficient. Additionally, the compression matched filter is greatly affected by the SNR. An algorithm for detecting signals in the sub-space with a low SNR was proposed in [33]. The author determined the subspace with a smaller dimension according to the known sparse characteristics of signals, and then designed and determined the measurement matrix to complete the detection of signals according to the properties of the subspace matrix. However, the algorithm fails to detect unknown sparse signals. In [35], Bayesian CS is studied in detail for signal detection, which needs the prior probability information of the sparse signal. However, when the background distribution characteristics of clutter are relatively complicated, it is difficult to detect the target in the clutter background [28]. Therefore, based on the combination of CD and CFAR detection, in this paper, a support vector data description (SVDD) is used to further classify the signal, which can further reduce the amount of data and improve the detection accuracy [36].

In this paper, the signal is not reconstructed after compression and detection, and the sparseness of the target in the distance cell in the radar echo signal is used to design a deterministic measurement matrix. Then, CFAR detection is directly performed to complete the first-order classification of the signal. This method has better detection performance than the compressive matched filter (CMF) algorithm in [32]. Compared with the traditional matched filtering (MF) algorithm, the data storage space is reduced under the premise of ensuring the detection probability. To address the problem of false alarms after first-order CFAR processing, the SVDD one-class classifier is used to train and classify the characteristic signal to achieve target detection through extraction of the feature difference between the target and the false alarm signal in the power spectrum. 


\section{System model descriptions}

\section{$2.1 \mathrm{CD}$ model}

From the theory of CS $[4,5]$, a discrete signal $x$ of length $L$ can be represented by a linear combination of a set of bases $\Psi \in\left[\psi_{1}, \psi_{2}, \cdots, \psi_{\mathrm{N}}\right]$

$$
\mathrm{x}=\sum_{i=1}^{N} \theta_{i} \psi_{i}=\Psi \theta
$$

in which $\boldsymbol{\theta} \in \boldsymbol{R}^{\mathrm{N} \times 1}$ is the sparse vector, $\theta_{i}=\left\langle x, \psi_{i}\right\rangle, x \in R^{\mathrm{L} \times 1}$ is the signal to be detected, and $\Psi^{\mathrm{L} \times \mathrm{N}}$ is the sparse basis or sparse dictionary. If $\theta$ has only $\mathrm{K}(\mathrm{K}=\mathrm{N})$ nonzero elements, then $\theta$ is termed the $\mathrm{K}$-sparse representation of the signal $x$. $\mathrm{K}$ is the sparsity of the signal.

When the signal $x$ can be sparsely represented [22], the problem of $\mathrm{CD}$ can be expressed by the following mathematical model of hypothesis testing

$$
\left\{\begin{array}{l}
\mathrm{H}_{0}: y=\Phi s_{n} \\
\mathrm{H}_{1}: y=\Phi\left(x+s_{n}\right)
\end{array}\right.
$$

in which $\Phi \in R^{\mathrm{M} \times \mathrm{N}}(\mathrm{M}=\mathrm{N})$ is the measurement matrix and $s_{n}$ is the Gaussian white noise. The hypothesis $\mathrm{H}_{0}$ is that the signal does not exist, and the hypothesis $\mathrm{H}_{1}$ is that the signal exists. Using Eq. (1), Eq. (2) can be rewritten as

$$
\left\{\begin{array}{l}
\mathrm{H}_{0}: y=\Phi s_{n} \\
\mathrm{H}_{1}: y=\Phi\left(\Psi \theta+s_{n}\right)
\end{array}\right.
$$

\subsection{Sparse representation model for radar signals}

Assuming that the radar target contains a number $\mathrm{K}$ of strong scattering centers located at different distance cells, the model of the pulse signal emitted $\mathrm{s}_{T}(t) \in \boldsymbol{R}^{L \times 1}$ by the radar is

$$
\boldsymbol{s}_{T}(t)=\operatorname{rect}\left(t / T_{P}\right) \exp \left(j 2 \pi f_{0} t+j \pi K_{r} t^{2}\right)
$$

The complex base-band echo signal received by the radar system $\boldsymbol{x}_{R}(\tau, t)$ can be described as

$$
\begin{gathered}
x_{\mathrm{R}}(\tau, \mathrm{t})=\sum_{\mathrm{k}=1}^{\mathrm{K}} \sigma_{\mathrm{k}} \exp \left[-\mathrm{j} 4 \pi \mathrm{f}_{0} \mathrm{r}_{\mathrm{k}}(\tau) / \mathrm{c}\right] \\
\cdot \operatorname{rect}\left[\left(\mathrm{t}-2 \boldsymbol{r}_{\mathrm{k}}(\tau) / \mathrm{c}\right) / \mathrm{T}_{\mathrm{P}}\right] \\
\cdot \exp \left\{j \pi \mathrm{K}_{\mathrm{r}}\left[\mathrm{t}-2 \boldsymbol{r}_{\mathrm{k}}(\tau) / \mathrm{c}\right]^{2}\right\} \\
+\boldsymbol{s}_{\mathrm{C}}(\mathrm{t})+\boldsymbol{s}_{\mathrm{n}}(\mathrm{t})
\end{gathered}
$$

in which $\sigma_{k}$ is the backscattering coefficient of the scattering point $k ; T_{P}$ is the time width of the pulse signal; $f_{0}$ is the center frequency of the signal; $r_{k}(\tau)$ is the distance between the scattering point $k$ and the radar platform at the moment of pulse emission; $c$ is the speed of light; $K_{r}$ is the frequency modulation coefficient; $s_{c}(t)$ is the clutter, which generally follows the Weibull distribution; and $\boldsymbol{s}_{n}(t)$ is the Gaussian white noise [37].

With $\boldsymbol{s}_{0}(t)=\left[\operatorname{rect}\left(t / T_{P}\right) /\left(T_{P}\left|K_{r}\right|\right)\right] \cdot \exp \left(j \pi K_{r} t^{2}\right)$ substituted into Eq. (5), the following is obtained 


$$
\begin{aligned}
\boldsymbol{x}_{R}(\tau, t)= & \sum_{k=1}^{K}\left\{\alpha_{k}(\tau) \boldsymbol{s}_{0}\left[t-2 r_{k}(\tau) / c\right]\right\} \\
& +\boldsymbol{s}_{c}(t)+\boldsymbol{s}_{n}(t) \\
= & \boldsymbol{s}_{r}(t)+\boldsymbol{s}_{c}(t)+\boldsymbol{s}_{n}(t)
\end{aligned}
$$

in which $\alpha_{k}(\tau)=T_{P}\left|K_{r}\right| \sigma_{\mathrm{k}} \exp \left[-\mathrm{j} 4 \pi \mathrm{f}_{0} \mathrm{r}_{\mathrm{k}}(\tau) / \mathrm{c}\right]$.

For the entire radar scene, the position occupied by the target is very small and is sparse for the total scanning area. The distance resolution of the radar in the observation interval $\left[r_{1}, r_{2}\right]$ is denoted $\Delta r$, and thus, the target scattering center in the distance cell can be represented by a one-dimensional vector $\theta$

$$
\theta^{\mathrm{T}}=\left[{ }_{1},{ }_{2}, \cdots,{ }_{1}, \cdots, \mathrm{L}-1_{1}, \mathrm{~L}\right]
$$

in which $\theta_{l}=\sigma_{l} \exp \left[-j 4 \pi f_{0} r_{l}(\tau) / c\right], \sigma_{l}$ is the backscattering coefficient of the scattering center of distance cell $r_{l}, r_{l}=r_{1}+l \Delta r, l \in[1: L]$, and $L=1+\left(r_{2}-r_{1}\right) / \Delta r$. When there is no target in a certain distance cell $k, \theta_{k}=0$. Through the delay of $s_{0}(t)$, the sparse dictionary basis $\Psi \in \boldsymbol{R}^{L \times N}$ of the radar signal is constructed:

$$
\begin{gathered}
\psi_{i}=\left[s_{0}\left(t_{i}-2 \frac{r_{1}}{c}\right) s_{0}\left(t_{i}-2 \frac{r_{1}+\Delta r}{c}\right)\right. \\
\cdots s_{0}\left(t_{i}-2 \frac{r_{1}+l \Delta r}{c}\right) \\
\left.\cdots s_{0}\left(t_{i}-2 \frac{r_{2}-\Delta r}{c}\right) s_{0}\left(t_{i}-2 \frac{r_{2}}{c}\right)\right]^{H} \\
=\left[s\left(t_{i}-\tau_{1}\right) s\left(t_{i}-\tau_{2}\right) \cdots s\left(t_{i}-\tau_{L}\right)\right]^{H}
\end{gathered}
$$

$\Psi=\left[\psi_{1}, \psi_{2}, \cdots, \psi_{N}\right]$, in which $i=1,2, \cdots, N$, and $\tau_{i}$ is the time delay corresponding to each distance cell. Given that the clutter and noise are not considered, according to Eqs. (1) and (8), the target scattering center can be sparsely expressed as

$$
\theta_{r}=\Psi^{\mathrm{H}} \boldsymbol{s}_{r}(t)
$$

\subsection{Measurement matrix model}

According to the theory of CS, with the complex base-band echo signal $\boldsymbol{s}_{r}(t)$ and through the design of the measurement matrix, the signal after $\mathrm{CD}$ can be directly detected. Let the measurement matrix be

$$
\Phi=\Phi_{1} \Psi^{\mathrm{H}}
$$

in which $\Phi_{1} \in \boldsymbol{R}^{M \times N}, N / M=l$, and $l$ is an integer. Then, $\Phi_{1}$ is defined as

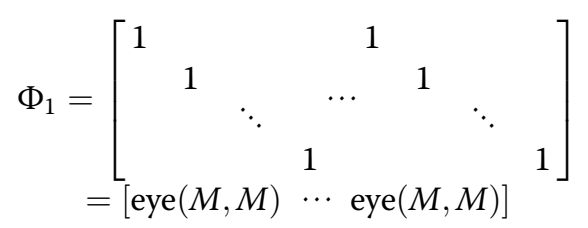

According to Eq. (3), the following is obtained

$$
\left\{\begin{array}{l}
H_{0}: \boldsymbol{y}=\Phi_{1} \Psi^{\mathrm{H}}\left(\boldsymbol{s}_{c}+\boldsymbol{s}_{n}\right) \\
H_{1}: \boldsymbol{y}=\Phi_{1} \Psi^{\mathrm{H}}\left(\boldsymbol{x}+\boldsymbol{s}_{c}+\boldsymbol{s}_{n}\right)
\end{array}\right.
$$

Substituting Eq. (11) into Eq. (12), the signal expression of hypothesis $H_{1}$ is obtained: 


$$
\begin{aligned}
& \boldsymbol{y}=\Phi_{1} \Psi^{\mathrm{H}}\left(\boldsymbol{x}+\boldsymbol{s}_{c}+\boldsymbol{s}_{n}\right) \\
& =\Phi_{1}\left(\theta_{r}+\boldsymbol{D}\right) \\
& =[\operatorname{eye}(M, M) \quad \cdots \quad \operatorname{eye}(M, M)] \\
& \left\{\left[\begin{array}{c}
0 \\
\vdots \\
\sigma_{1}^{2} \\
\vdots \\
0 \\
\sigma_{L}^{2} \\
\vdots \\
0
\end{array}\right]+\left[\begin{array}{c}
\boldsymbol{D}(1) \\
\boldsymbol{D}(2) \\
\\
\vdots \\
\boldsymbol{D}(N-1) \\
\boldsymbol{D}(N)
\end{array}\right]\right\}
\end{aligned}
$$

Therefore, $\boldsymbol{D}=\Psi^{\mathrm{H}}\left(\boldsymbol{s}_{c}+\boldsymbol{s}_{n}\right)$. Since the radar target signal $\boldsymbol{x}$ is sparse, Eq. (13) shows that $\sigma_{1}^{2}, \cdots, \sigma_{L}^{2}$ are $L$ targets' projections on the dictionary basis. When the $i$-th line (1 $\leq$ $i \leq L$ ) element 1 of $\Phi_{1}$ is multiplied by $\sigma_{i}^{2}$ and $\boldsymbol{D}$, the results are all 0 except $\boldsymbol{D}$ multiplied by the $i$-th element 1 of $\Phi_{1}$. Good noise reduction performance is thus achieved. The noise reduction performance increases with increasing $M$. Through the $C D$ of the echo signal, the sampling rate is reduced. Additionally, a storage space savings of $1 / l$ is achieved, and the direct detection of the compressed signal is realized without reconstruction.

\subsection{SVDD model}

Currently, the classifier is divided into a one-class classifier and a multiclass classifier according to the number of categories of training samples [38]. The multiclass classifiers are used mainly for classification problems where the training samples are sufficient and the data of different categories are relatively balanced. Different types of training samples are needed to construct the classification function, and the sample classification is achieved by determining the optimization points between different categories. A one-class classifier is mainly used in scenarios where multiple types of training samples cannot be obtained or are too expensive to obtain. This classifier needs a training sample from only one target category. The training sample of this category is used to construct a closed cover. The unknown test sample is determined as a target or non-target. An SVDD is often used in the field of abnormal data detection and is a very typical classifier. In the training phase, training samples from only one type of target are needed to obtain the optimal classification surface [36]. Assuming that the radius of the hypersphere found in the high-dimensional feature space is $R_{\phi}$ and that the sphere center vector of the hypersphere is $a_{\phi}$, the expression for any point on the hypersphere $\phi\left(x_{i}\right)$ is $\left\|\phi\left(x_{i}\right)-a_{\phi}\right\|^{2}=R_{\phi}^{2}$. Therefore, the solution process for the optimal classification surface of the SVDD can be expressed as

$$
\left\{\begin{array}{l}
\min \mathrm{R}_{\phi}^{2}+\mathrm{C} \sum_{i=1}^{l} \xi_{i} \\
\text { s.t. }\left\|\phi\left(x_{i}\right)-a_{\phi}\right\|^{2} \leq \mathrm{R}_{\phi}^{2}+\xi_{i}, \xi_{i} \geq 0, i=1,2, \cdots, l
\end{array}\right.
$$

in which $l$ is the number of sample points, $C$ is the penalty coefficient, and $\xi_{i}$ is the Lagrange multiplier corresponding to the $i$-th sample. With the Lagrange multiplier method, we can obtain the dual optimization problem 


$$
\left\{\begin{array}{l}
\max \sum_{i=1}^{l} \beta_{i} \mathrm{~K}\left(\mathrm{x}_{i}, \mathrm{x}_{j}\right)-\sum_{i=1}^{l} \sum_{j=1}^{l} \beta_{i} \beta_{j} \mathrm{~K}\left(\mathrm{x}_{i}, \mathrm{x}_{j}\right) \\
\text { s.t. } \sum_{i=1}^{l} \beta_{i}=1, \beta_{i} \in[0, C], i=1,2, \cdots, l
\end{array}\right.
$$

in which the kernel function $K\left(x_{i}, x_{j}\right)=\phi^{\mathrm{T}}\left(x_{i}\right) \phi\left(x_{i}\right)$ and $\beta_{i}$ is the Lagrange multiplier. In the process of solving the dual optimization problem, the expression of the sphere center vector of the hypersphere is obtained:

$$
a_{\phi}=\sum_{i=1}^{l} \beta_{i} \phi\left(x_{i}\right)
$$

With the Lagrange multiplier and any support vector on the hypersphere $\phi(x)$, the radius of the hypersphere can be obtained:

$$
\begin{aligned}
R_{\phi} & =K(x, x)-2 \sum_{i=1}^{l} \beta_{i} K\left(x_{i}, x\right) \\
& +\sum_{i=1}^{l} \sum_{j=1}^{l} \beta_{i} \beta_{j} K\left(x_{i}, x_{j}\right)
\end{aligned}
$$

Any sample point $x$ becomes $\phi(x)$ after being mapped to the high-dimensional feature space, and the decision-making method is

$$
f_{\phi}(x)=\left\|\phi(x)-a_{\phi}\right\|^{2}-R_{\phi}^{2}
$$

When $f_{\phi}(x) \leq 0, x$ is the target sample. Otherwise, $x$ is an abnormal sample.

\section{Two-stage classification algorithm}

\subsection{Algorithm flow}

The traditional CFAR detectors mainly include CA-CFAR, ordered statistic CFAR (OSCFAR), etc. [39] CA-CFAR, which is the most widely used CFAR detection algorithm, detects whether or not the signal exists by comparing the arithmetic mean values of the measured cell and several adjacent reference cells. OS-CFAR sorts the data in the reference cell, then selects the value of the $k$-th element as an estimate of cell's the clutter level and multiplies this value by a scale factor $T$ as the detection threshold to detect whether or not the signal exists. In addition, we still need to establish how to set the detection threshold, which references $[25,39]$ show how to do. In the CA-CFAR detector, the estimate of the background clutter power level is the sum of $2 n$ reference cells.

$$
Z=\sum_{i=1}^{2 n} \boldsymbol{y}_{i}
$$

The CFAR condition can be obtained by multiplying the scale factor $T$ by the estimated background level. The detection probability of the CA-CFAR detector is

$$
P_{d}=\left(1+\frac{T}{1+S}\right)^{-2 n}
$$


Here, $T$ is a scale factor, and $S$ is the average SNR. In the OS-CFAR detector, the $2 n$ reference cell signals are sorted by amplitude $\left(y_{1} \leq y_{2} \leq \cdots \leq y_{k} \leq \cdots \leq y_{2 n}\right)$; the $k$-th value is taken as the background noise estimate:

$$
Z=y_{k}
$$

The detection probability of OS-CFAR can be expressed as

$$
P_{d}=\prod_{i=0}^{k-1} \frac{2 n-i}{2 n-i+T /(1+S)}
$$

The CFAR models used in this algorithm are CA-CFAR and OS-CFAR, and the classifier is the SVDD one-class classifier. Figure 1 shows the detection scheme of the twostage classification algorithm. The overall algorithm is divided into two stages.

The first stage of classification:

Step 1: Design the sparse dictionary basis according to the complex base-band echo signal, and then design the measurement matrix $\Phi=\Phi_{1} \Psi^{\mathrm{H}}$ with Eq. (10);

Step 2: Compress and detect the echo signal by the measurement matrix of Eq. (10) to obtain Eq. (12) $y$;

Step 3: Send the CD signal $\boldsymbol{y}$ to the CFAR detector, and perform CA-CFAR or OS-

CFAR processing on the $2 n$ data except for the measured cell to obtain the result $Z$. If the signal is greater than the threshold $(Y>T Z)$, the state is $H_{1}$, namely, the target exists; otherwise, the state is $H_{0}$, and the target does not exist.

The second stage of classification (divided into training section and test section):

Step 4: Take the non-target signal $\left(\mathrm{H}_{0}\right)$ in the first stage as the training data, extract the power spectrum feature of each group of training samples to form a feature vector,

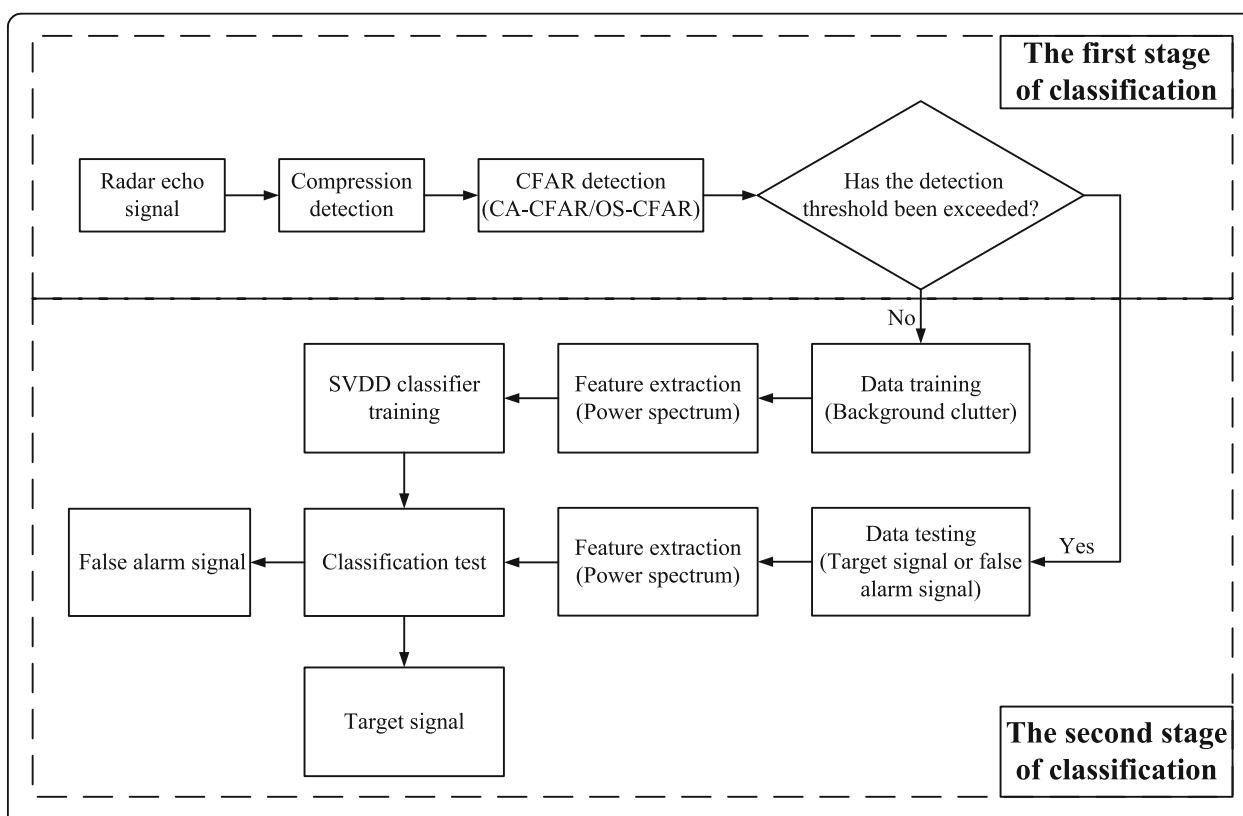

Fig. 1 Detection scheme of the two-stage classification algorithm 
and form the feature vector matrix using the feature vectors of all training samples.

Then, train the SVDD classifier;

Step 5: Use the target or false alarm echo data $\left(\mathrm{H}_{1}\right)$ in the first stage as the test data, then extract the power spectrum feature for each set of test samples, send the feature vector to the trained SVDD classifier, and complete the target detection.

Through the two-stage classification algorithm for radar targets, after the radar echo signal is compressed and detected in the first stage after the CFAR processing, the signal is divided into background clutter and target signals (including false alarm signals). In the second stage, the power spectrum feature is used to perform SVDD classification and to achieve classification of the target signals and false alarm signals.

\subsection{Power spectrum feature extraction}

For radar echo signals, the power spectrum of the signal can be obtained by using the fast Fourier transform. In the SVDD classifier, the power spectrum of the radar echo signal is extracted as a feature vector for target algorithm classification. Since the power spectrum is high-dimensional data, extensive calculations will be needed if the power spectrum is directly used as a feature vector. Therefore, in this paper, dimensionality reduction is performed on the power spectrum feature vector, and the two-dimensional features are extracted; that is, the second-order central moment of the power spectrum and the power spectrum entropy are used to describe the dispersion characteristics of the power spectrum, which is used as a feature vector. Let the radar echo signal be $\boldsymbol{x}=$ $\left[x_{1}, x_{2}, \cdots, x_{K}\right]$. Then, the frequency spectrum is $\boldsymbol{F}=\operatorname{FFT}(\boldsymbol{x})=\left[\boldsymbol{F}_{1}, \boldsymbol{F}_{2}, \cdots, \boldsymbol{F}_{K}\right]^{\mathrm{T}}$, and the power spectrum is $\boldsymbol{p}=|\boldsymbol{F}|^{2}=\left[p_{1}, p_{2}, \cdots, p_{K}\right]^{\mathrm{T}}$, where $K$ is the dimension of the signal.

Feature 1: The second-order central moment of the power spectrum is

$$
\mathrm{S}_{1}=\sum_{k=1}^{K}(k-\tilde{k})^{2} b_{k}
$$

in which $b_{k}=p_{k} / \sum_{k=1}^{K} x_{k}$ and $\tilde{k}=\sum_{k=1}^{K} k \times b_{k}$. In the random process, the secondorder central moment describes the distribution of the power spectrum energy distribution relative to its geometric centroid. If the second-order central moment is smaller, the energy distribution is more scattered relative to the centroid.

Feature 2: The power spectrum entropy is

$$
\mathrm{S}_{2}=-\sum_{k=1}^{K} b_{k} \lg \left(b_{k}\right)
$$

The power spectrum entropy is used to describe the energy distribution characteristics of the power spectrum. The more concentrated the power spectrum energy distribution is, the lower the entropy.

\section{Experiment results and discussion}

\subsection{First-order target classification experiment}

In this section, simulation experiments are conducted on two kinds of radar echo in 15 $\mathrm{km}$ scenarios (a single-target radar echo scenario and a multi-target radar echo scenario) and compared with the performance of the traditional MF and CMF [32] 
detection algorithms to evaluate the detection performance of the proposed algorithm. In all the simulations, the radar echo signal is

$$
\boldsymbol{s}_{r}(t)=\sum_{i=1}^{m} \exp \left(j \pi \mathrm{K}_{r}\left(t-\tau_{i}\right)^{2}\right)+\boldsymbol{s}_{c}(t)+\boldsymbol{s}_{n}(t)
$$

in which $m$ is the number of targets, $\tau_{i}$ is the time delay of the $i$-th target, $\boldsymbol{s}_{c}(t)$ is the Weibull background clutter, and $\boldsymbol{s}_{n}(t)$ is the Gaussian white noise.

In Experiment 1, the minimum detection distance of the radar was $R_{\min }=5000 \mathrm{~m}$, the maximum detection distance was $R_{\max }=15000 \mathrm{~m}$, the pulse width was $B=30 \mathrm{MHz}$, the frequency modulation slope was $K_{r}=B / T=3 \times 10^{12} \mathrm{~Hz} / \mathrm{s}$, the sampling frequency was $f_{s}=150 \mathrm{MHz}$, and a single target location was set at $R=9000 \mathrm{~m}$. In Experiment 2, four target positions were set at $R=[6000650070008000] \mathrm{m}$, and the sparse dictionary base $\Psi$ is designed based on Eq. (8). Fig. 2 is the detection effect of the algorithm on the direct $\mathrm{CD}$ of CFAR for a single-target radar echo signal. Fig. 3 represents the detection effect of the algorithm on the direct CD of CFAR for multi-target radar echo signals. Fig. 4 compares the performances of the algorithm in this paper, the traditional MF detection algorithm, and the CMF algorithm [32]. Fig. 5 compares the detection performances of the three detection algorithms in CA-CFAR and OS-CFAR. The variation curve of the detection probability with the SNR ratio and different compressive ratios over 1,000 Monte Carlo experiments is depicted in Fig. 6.

Figure 2 compares the $\mathrm{CD}$ signal and the CFAR detection threshold for SNR = $10 \mathrm{~dB}$, a signal-to-clutter ratio $\mathrm{SCR}=-10 \mathrm{~dB}, M / N=0.5$. For a low $\mathrm{SNR}$, the direct compression and detection of the signal can still clearly determine the target position without reconstruction of the signal.

Figure 3 compares the multi-target $\mathrm{CD}$ signal and the CFAR detection threshold for $\mathrm{SNR}=-10 \mathrm{~dB}, \mathrm{SCR}=-10 \mathrm{~dB}$, and $M / N=0.5$. With four targets, in the case of a low SNR and a low SCR, the first-order classification of the target can be completed through CFAR detection.

Figure 4 compares the detection performances of the algorithm in this paper, the traditional MF algorithm and the CMF algorithm. Under the same compressive ratio,

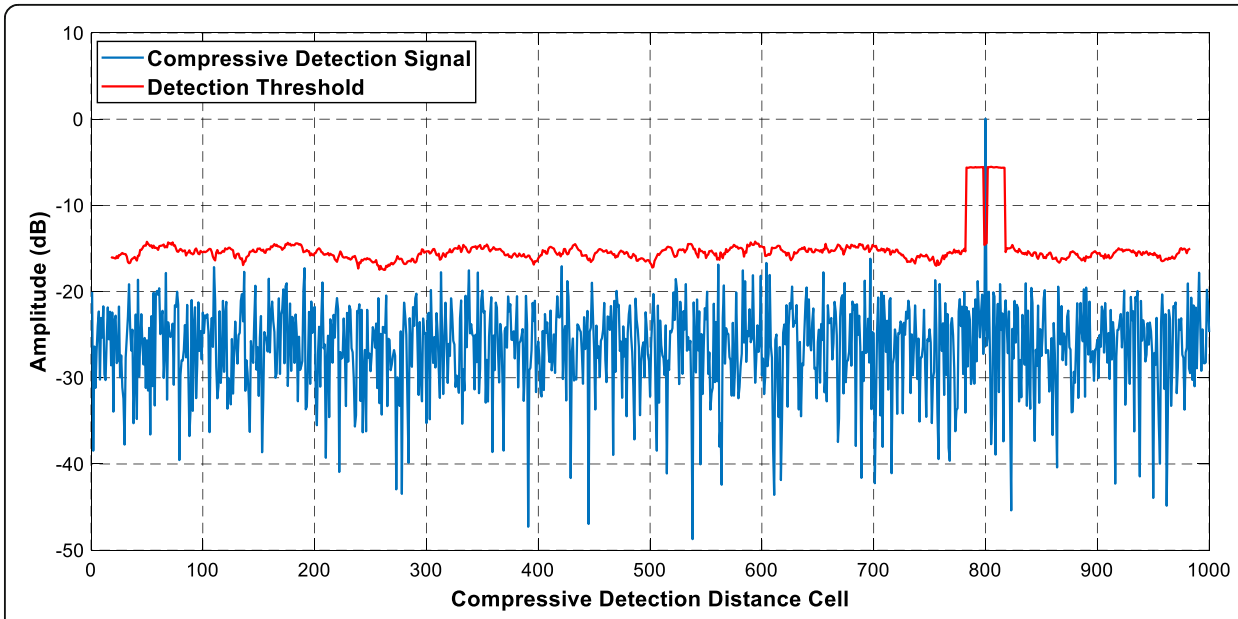

Fig. 2 Comparison between single target radar echo signal CD and CFAR detection threshold 


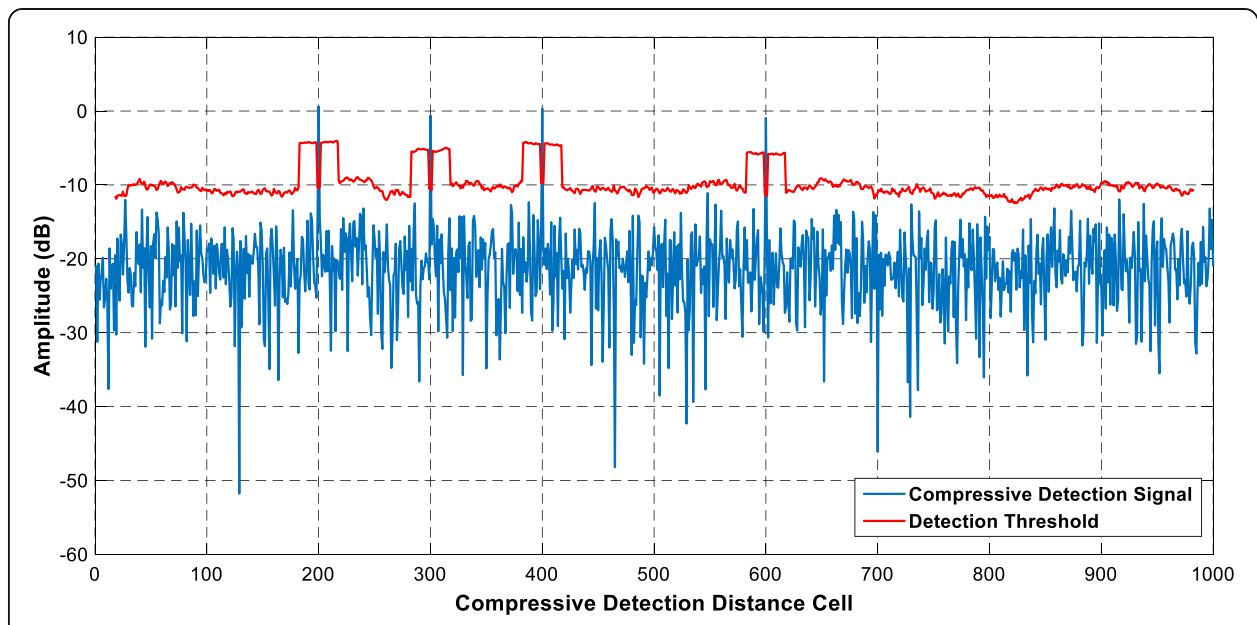

Fig. 3 Comparison between multi-target radar echo signal CD and CFAR detection threshold

the detection performance of the algorithm in this paper is significantly better than that of the CMF algorithm (Fig. 4). When SNR $=-15 \mathrm{~dB}$, the detection probability of the algorithm in this paper reaches $96 \%$; this detection performance is close to that of the traditional MF algorithm, while its data storage requirement is reduced by half.

Figure 5 illustrates a comparison of the detection performance of the three detection algorithms in CA-CFAR and OS-CFAR, where $\mathrm{SCR}=-10 \mathrm{~dB}, M / N=0.5$, and the false alarm probability $P_{\mathrm{f}}=10^{-3}$. With the same false alarm probability, the detection performance of CA-CFAR and OS-CFAR is similar. Additionally, the algorithm in this paper reduces the data calculations to half of those of the traditional MF algorithm.

Figure 6 gives the detection probabilities for different compressive ratios with the SNR when $\mathrm{SCR}=-10 \mathrm{~dB}$ and the false alarm probability $P_{\mathrm{f}}=10^{-3}$. Under the same $\mathrm{SNR}$, the detection probability of the signal increases as the compressive ratio increases. When the $\mathrm{SNR}=-12 \mathrm{~dB}$ and $M / N=0.25$, the target detection probability reaches $95 \%$.

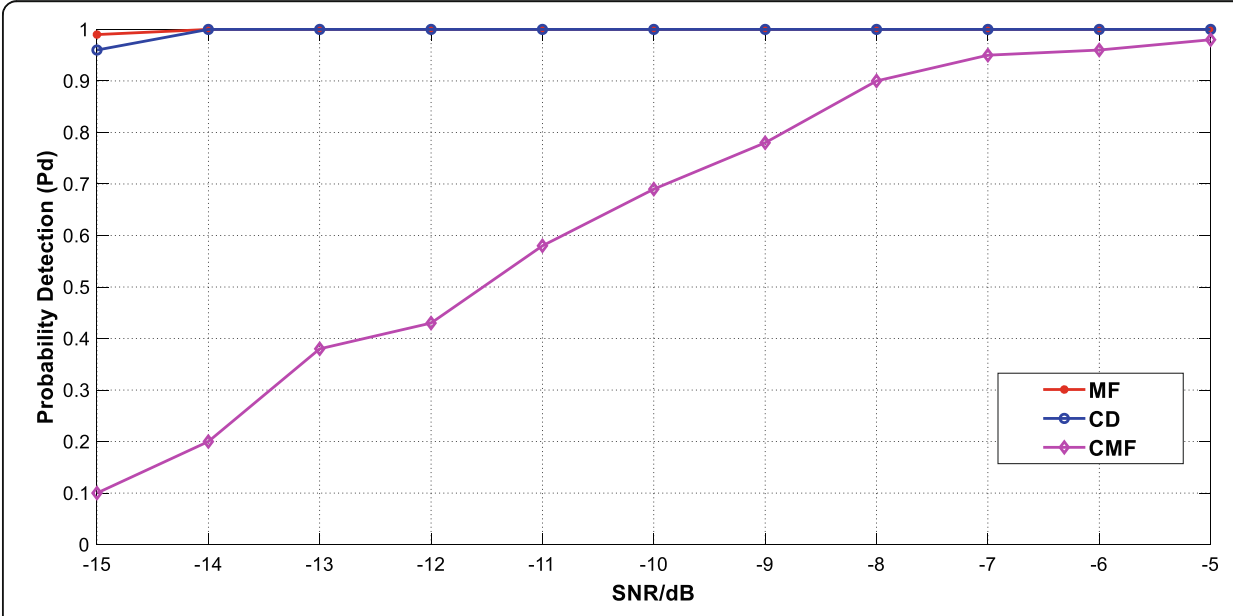

Fig. 4 Comparison of the detection performance of the three detection algorithms 


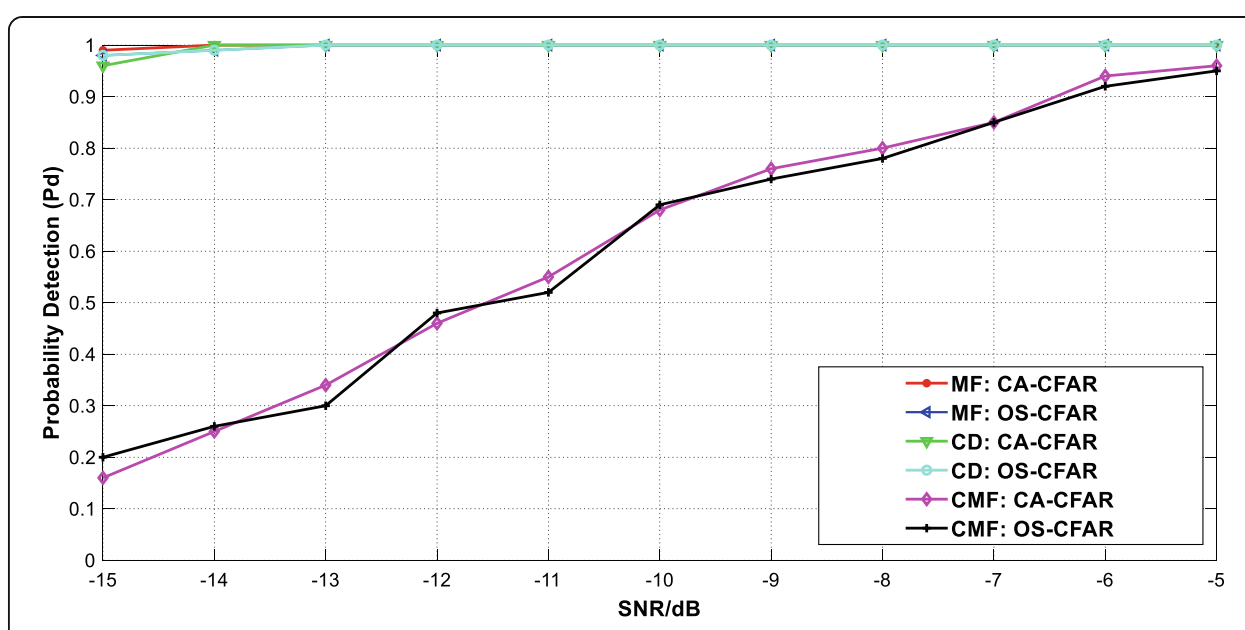

Fig. 5 Comparison of the detection performance of the three detection algorithms in CA-CFAR and OS-CFAR

\subsection{Two-stage target classification experiment}

Since radar targets are usually non-cooperative, there are usually no target training samples or false alarm signals available in advance, and only targets such as background clutter are used as training samples. Therefore, the SVDD one-class classifier is selected to classify the targets. In this section, the first-stage target after CD of CFAR is further classified by the SVDD one-class classifier to differentiate targets and false alarm signals. For the second-stage classification experiment, the signal is set as $\boldsymbol{x}=\left[x_{1}, x_{2}, \cdots\right.$, $x_{K}$, the signal dimension $K=200$, and the power spectrum features of the signal are extracted. Figures 7(a) and 7(b) show the average power spectra (APS) of the background clutter, false alarm signals, and target signals in the first-stage target classification experiment.

The target signal exhibits obvious feature differences from the background clutter and false alarm signal in the power spectrum domain (Fig. 7). The overall distribution trends of the power spectra of the three signals show that the shape of the power spectrum curve of the target signal changes relatively smoothly. However, the power spectrum curves of the false alarm signal and the background clutter change more

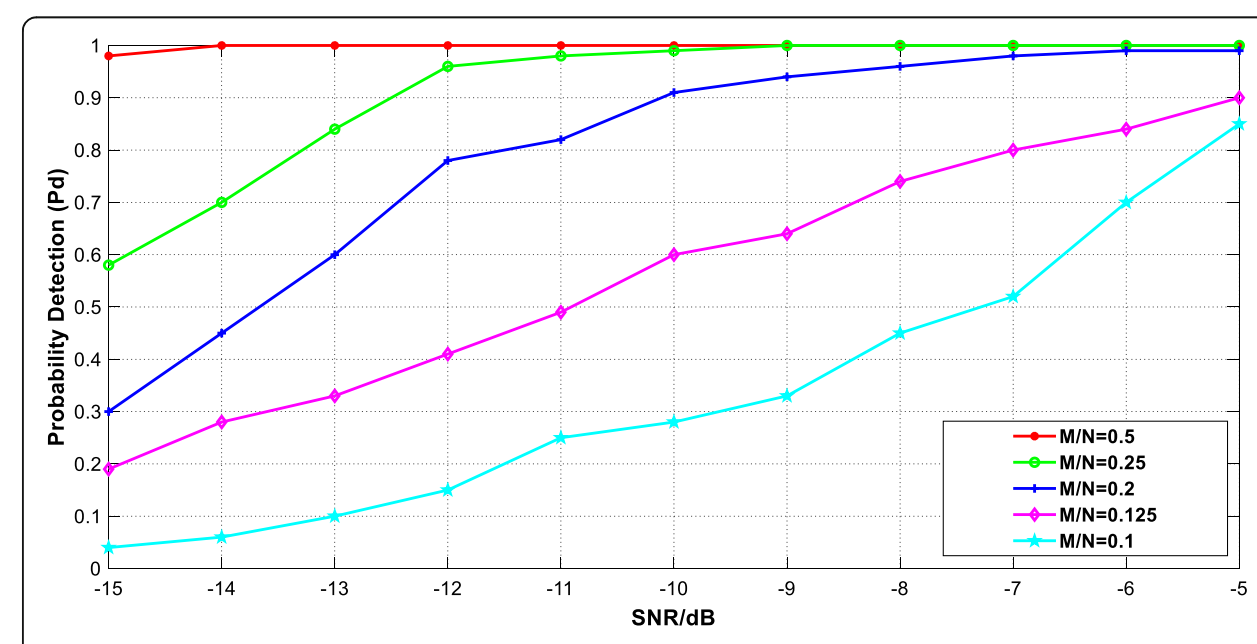

Fig. 6 CFAR detection probability under different compressive ratios 

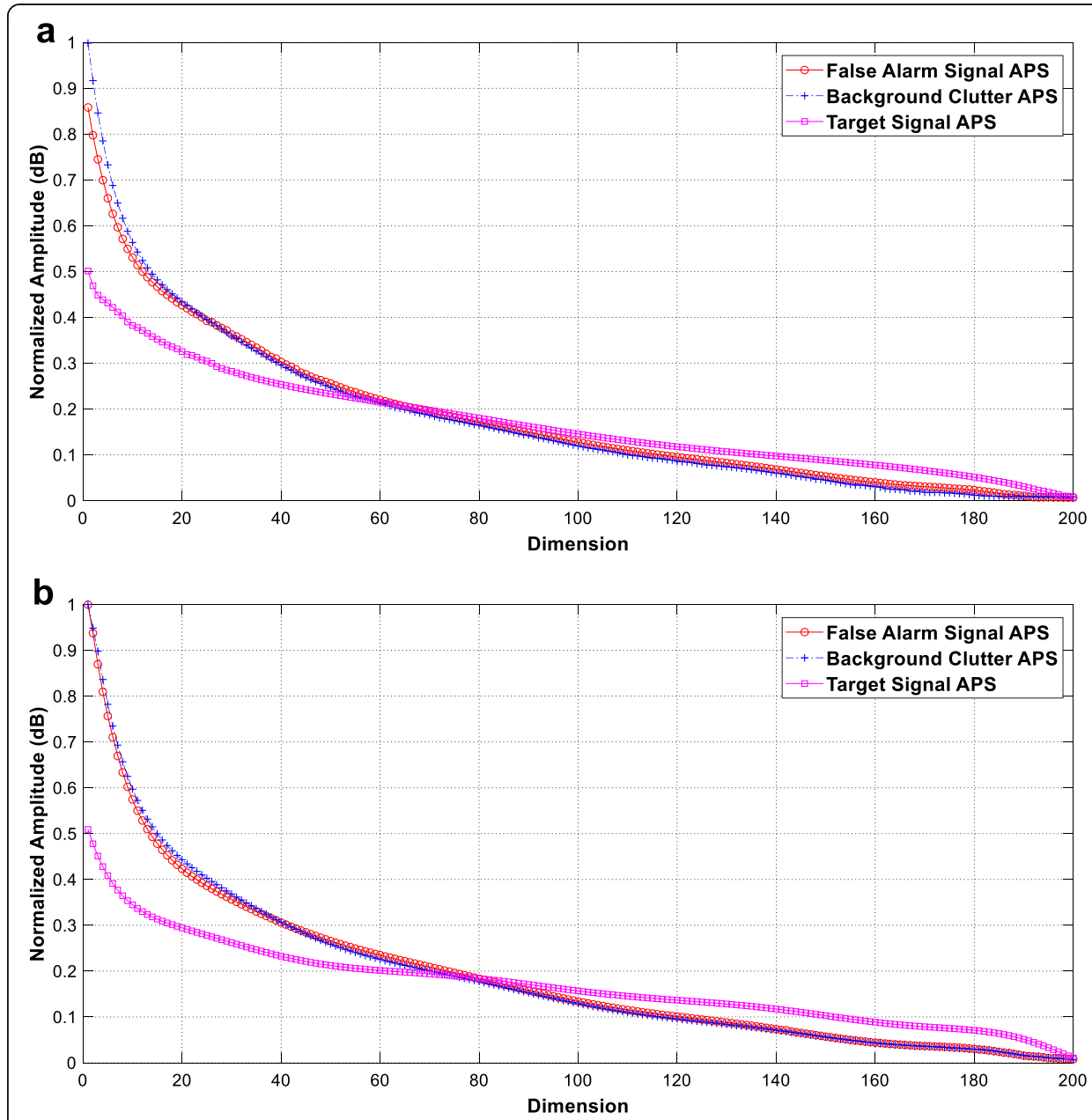

Fig. 7 APS of the background clutter, the false alarm signal, and the target signal. a Power spectrum of single target scene. $\mathbf{b}$ Power spectrum of multi-target scene

quickly, and their distribution characteristics are very similar and consistent, which is obviously different from that of the target signal. The power spectrum of the target signals in Fig. 7 is more even than its centroid distribution, and the second-order central moment of its power spectrum is larger than the second-order central moments of the false alarm signals and background clutter. From an energy perspective, the power spectrum energy distribution of the target signals is more even and its entropy is greater than those of the false alarm signals and background clutter.

For a given training set that contains $n$ target samples, $X=\left\{x_{1}, x_{2}, \cdots, x_{n}\right\}$, the goal of SVDD is to find a hypersphere with the smallest volume in a high-dimensional space so that all target samples or as many as possible are enclosed in the hypersphere instead of the scenario where the target sample is as small as possible or none of the target samples are in the hypersphere. In this experiment, according to the SVDD model, the Gaussian kernel function is selected. After the feature vector of the background clutter is selected as the training data, the SVDD one-class classifier is trained to obtain the optimal classification surface. Figures $8(\mathrm{a})$ and 8 (b) show the results of using the SVDD optimal classification to classify the test sample during the test phase. In addition to the background clutter in the training sample, false alarm signals are 
present in the test sample that are determined to be background clutter inside the classification surface. The test samples outside the classification surface are determined to be the target signals.

The SVDD experiment results show that the target signals are all outside the optimal classification surface and that the classification results are correct (Fig. 8). All of the false alarm signals are included inside the optimal classification surface and are determined to be background clutter. In single-target and multi-target scenarios, the proposed algorithm can effectively reduce the number of false alarms and increase the probability of signal detection. In the radar target detection process, false alarms usually increase the detection workload, but a missed alarm can cause a serious accident. The algorithm in this paper has good adaptability to such situations. The amount of data storage is reduced, and the number of false alarms can be effectively lowered.

\section{Conclusions}

In this paper, the CD CFAR is used and combined with an SVDD classifier to form a two-stage classification algorithm for radar target detection. In the distance domain, through the design of the measurement matrix, the radar echo signal is directly compressed and detected without reconstruction of the signal, and the first-stage classification of the radar echo signal is then completed through the CFAR detection structure. To separate false alarm signals and target signals after the first stage of classification, the SVDD is introduced as the second stage of classification. Features of the signal power spectrum are extracted after compression and detection of CFAR, and the SVDD one-class classifier is used in the feature domain. The background clutter is used as training data to complete the classification of false alarm signals and target signals. The analysis of the experimental results shows that the algorithm in this paper can still detect the target position under the conditions of a low SNR and a low SCR. Additionally, under the premise of ensuring a high detection probability, the necessary data storage space is reduced. The detection performance is improved, the number of false alarms is effectively lowered, and good adaptability is maintained.

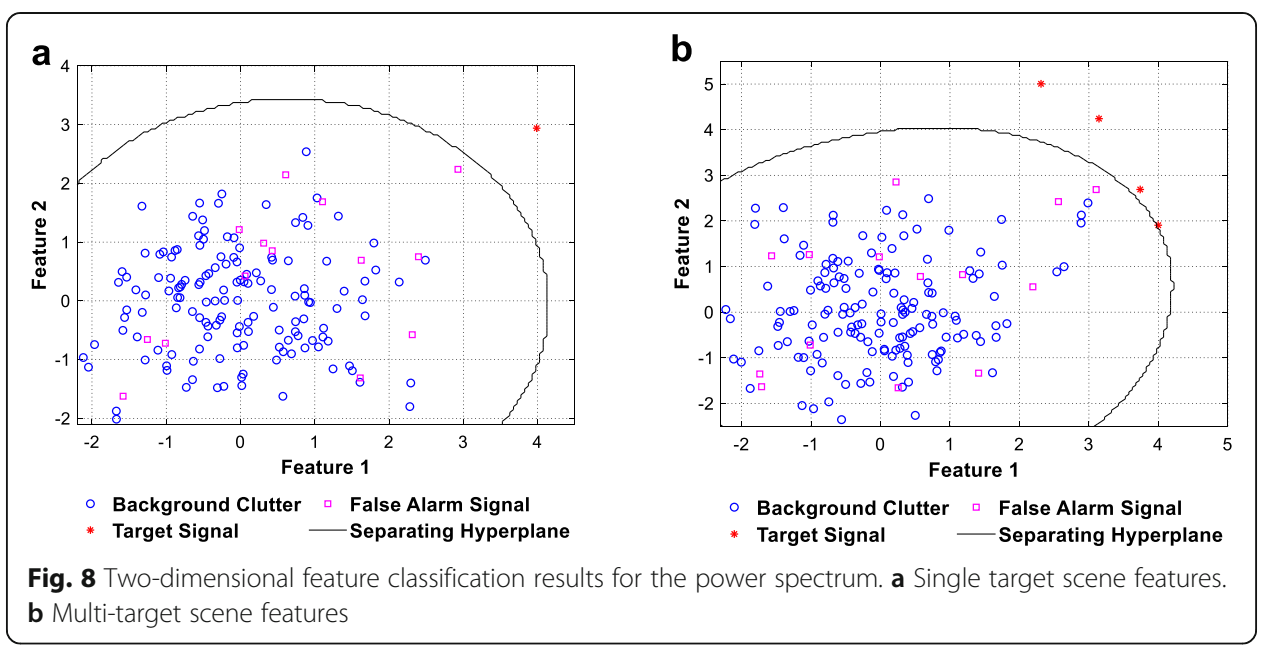




\section{Abbreviations}

CS: Compressed sensing; CD: Compressive detection; SNR: Signal-to-noise ratio; SCR: Signal-to-clutter ratio; SVDD: Support vector data description; CFAR: Constant false alarm rate; CA-CFAR: Cell average CFAR; OSCFAR: Ordered statistic CFAR; MF: Matched filtering; CMF: Compressive matched filter; APS: Average power spectra

\section{Acknowledgements}

Not applicable.

\section{Authors' contributions}

$\mathrm{CL}$ proposed the framework of the whole algorithm; performed the simulations, analysis and interpretation of the results; and drafted the manuscript. YL acquired funding. XL and QZ have participated in the conception and design of this research. The paper was revised by TW and QL. All authors read and approved the final manuscript.

\section{Funding}

This work was supported by the Science and Technology Department Project of Jilin Province (under grants No. 20190303034SF and No. 20180201064GX).

\section{Availability of data and materials}

Please contact the authors for data requests.

\section{Ethics approval and consent to participate}

Not applicable.

\section{Consent for publication}

The manuscript does not contain any individual person's data in any form (including individual details, images, or videos), and therefore, the consent to publish is not applicable to this article.

\section{Competing interests}

The authors declare that they have no competing interests.

Received: 24 October 2020 Accepted: 25 January 2021

Published online: 20 May 2021

\section{References}

1. K. Christina, S. Benedikt, S. Susanne, et al., High range and Doppler resolution by application of compressed sensing using low baseband bandwidth OFDM radar. IEEE Trans. Microw. Theory Tech. 32(7), 1-12 (2018)

2. C. David, C. Deborah, Y.C. Eldar, et al., SUMMeR: Sub-Nyquist MIMO radar. IEEE Trans. Signal Process. 66(16), 4315-4330 (2018)

3. I. Taghavi, M.F. Sabahi, F. Parvaresh, High resolution compressed sensing radar using difference set codes. IEEE Trans. Signal Process. 61(1), 136-148 (2019)

4. D.L. Donoho, Compressed sensing. IEEE Trans. Inf. Theory 52(4), 1289-1306 (2006)

5. E.J. Candes, T. Tao, Near-optimal signal recovery from random projections: Universal encoding strategies. IEEE Trans. Inf. Theory 52(12), 5406-5424 (2006)

6. B.Y. Liu, Y. Zhao, X.M. Zhu, et al., Sparse detection algorithms based on two-dimensional Compressive sensing for subNyquist pulse Doppler radar systems. IEEE Access. 7, 18649-18661 (2019)

7. Y. Sun, J.J. Huang, J.X. Huang, et al., Compressive detection using sub-Nyquist radars for sparse signals. Int J Antennas Propagation 2016, 1-7 (2016)

8. C. Knill, F. Roos, B. Schweizer, et al., Random multiplexing for an MIMO-OFDM radar with compressed sensing-based reconstruction. Microw. Wireless Components Lett. 29(4), 300-302 (2019)

9. F. Zhong, S.X. Guo, X.X. Joint Channel, Coding based on LDPC codes with Gaussian kernel Reflecton and CS redundancy. Appl. Math. Inf. Sci. 7(6), 2421-2425 (2013)

10. E.J. Candes, M.B. Wakin, An introduction to Compressive sampling. IEEE Signal Process. Mag. 25(2), 21-30 (2008)

11. W. Dai, O. Milenkovic, Subspace pursuit for Compressive sensing signal reconstruction. IEEE Trans. Inf. Theory 55(5), 2230-2249 (2009)

12. T. Blumensath, M.E. Davies, Iterative hard Thresholding for compressed sensing. Appl. Comput. Harmon. Anal. 27(3), 265-274 (2009)

13. J.A. Tropp, A.C. Gilbert, Signal recovery from partial information via orthogonal matching pursuit. IEEE Trans. Inf. Teory. 53(12), 4655-4666 (2006)

14. S.R. Chai, L.X. Guo, K. Li, et al., Fast analysis of multi-static scattering problems with Compressive sensing technique. J. Quant. Spectrosc. Radiat. Transf. 202, 136-146 (2017)

15. V.D.B. Ewout, M.P. Friedlander, Probing the Pareto frontier for basis pursuit solutions. SIAM J. Sci. Comput. 31(2), 890-912 (2008)

16. S.D. Babacan, R. Molina, A.K. Katsaggelos, Bayesian Compressive sensing using Laplace priors. IEEE Trans. Image Process. 19(1), 53-64 (2010)

17. K. Gkoktsi, A. Giaralis, A Compressive MUSIC spectral approach for identification of closely-spaced structural natural frequencies and post-earthquake damage detection. Probabilistic Eng. Mech. 60, 103030 (2020)

18. Z. Zhang, B.D. Rao, Sparse signal recovery with temporally correlated source vectors using sparse Bayesian learning. IEEE J. Selected Top. Signal Process. 5(5), 912-926 (2011)

19. M.A. Davenport, P.T. Boufounos, M.B. Wakin, Signal processing with Compressive measurement. IEEE J. Selected Top. Signal Process. 4(2), 445-460 (2010) 
20. Y. Wang, Z. Liu, L. Yang, et al., Generalized Compressive detection of stochastic signals using Neyman-Pearson theorem. Signal Image Video Process. 9, 111-120 (2015)

21. S. Qin, D. Chen, X. Huang, et al., A Compressive signal detection scheme based on Sparsity. International journal of signal processing. Image processing and. Pattern Recogn. 7(2), 121-130 (2014)

22. A. Hariri, M. Babaie-Zadeh, Joint Compressive single target detection and parameter estimation in radar without signal reconstruction. IET Radar. 9(8), 948-955 (2015)

23. T. Wimalajeewa, P.K. Varshney, Sparse signal detection with Compressive measurements via partial support set estimation. IEEE Trans. Signal Inf. Process. Over Netw. 3(1), 46-60 (2017)

24. C. Hu, J.Y. Kim, S.Y. Na, Compressive frequency hopping signal detection using spectral kurtosis and residual signals. Wirel. Pers. Commun. 94(1), 53-67 (2017)

25. H. Rohling, Radar CFAR Thresholding in clutter and multiple target situations. IEEE Trans. Aerosp. Electron. Syst. 19(4), 608-621 (1983)

26. W. Jiang, Y.L. Huang, J.Y. Yang, Automatic censoring CFAR detector based on ordered data difference for low-flying helicopter safety. Sensors. 16(7), 1055-1075 (2016)

27. N. Fatih, E.O. Osman, O. Atilla, et al., RmSAT-CFAR: Fast and accurate target detection in radar images. SoftwareX. 8, 3942 (2018)

28. F. Wang, X.B. Cong, C.G. Shi, et al., Target tracking while jamming by airborne radar for low probability of detection. Sensors. 18(9), 2903-2919 (2018)

29. D. Barchiesi, M.D. Plumbley, Learning incoherent dictionaries for sparse approximation using iterative projections and rotations. IEEE Trans. Signal Process. 61(8), 2055-2065 (2013)

30. L. Anitori, W.V. Rossum, M. Otten, et al., Proc. 2016 IEEE Radar Conference (RadarConf). Design of CFAR Radars Using Compressive Sensing (IEEE express Conference Publishing, Philadelphia, 2016), pp. 1-6

31. L. Anitori, M. Otten, A. Maleki, IEEE Radar Conference. Compressive CFAR Radar Detection (IEEE express Conference Publishing, Atlanta, 2012) (2012), pp. 0320-0325

32. M.A. Davenport, M.B. Wakin, R.G. Baraniuk, in Technical Report TREE, 0610, Rice University. The Compressive matched filter. Rice University (2006)

33. A. Razavi, M. Valkama, D. Cabric, Compressive detection of random subspace signals. IEEE Trans. Signal Process. 64(16), 4166-4179 (2016)

34. A. Hariri, M. Babaie-Zadeh, Compressive detection of sparse signals in additive white Gaussian noise without signal reconstruction. Signal Process. 131, 376-385 (2016)

35. M. Başaran, S. Erküçük, H.A. Çırpan, Bayesian Compressive sensing for primary user detection. IET. Signal Process. 10(5), 514-523 (2016)

36. D.M.J. Tax, R.P.W. Duin, Support vector data description. Mach. Learn. 54(1), 45-66 (2004)

37. T.Y. Huang, Y.M. Liu, X.Y. Xu, et al., Analysis of frequency agile radar via compressed sensing. IEEE Trans. Signal Process. 66(23), 6228-6240 (2018)

38. V.K. Chauhan, K. Dahiya, A. Sharma, Problem formulations and solvers in linear SVM: A review. Artif. Intell. Rev. 52(2), 803-855 (2018)

39. S.W. Hong, D.S. Han, Performance analysis of an environmental adaptive CFAR detector. Math. Probl. Eng. 2014(6), 1-7 (2014)

\section{Publisher's Note}

Springer Nature remains neutral with regard to jurisdictional claims in published maps and institutional affiliations.

\section{Submit your manuscript to a SpringerOpen ${ }^{\circ}$ journal and benefit from:}

- Convenient online submission

- Rigorous peer review

- Open access: articles freely available online

- High visibility within the field

- Retaining the copyright to your article

Submit your next manuscript at $\boldsymbol{\Delta}$ springeropen.com 